\title{
Multi-angle View, Illumination and Cosmetic Facial Image Database (MaVIC) and Quantitative Analysis of Facial Appearance
}

\author{
Yen-Wei Chen ${ }^{1}$, Takayuki Fukui ${ }^{1}, \mathrm{Xu}_{\text {Qiao }}{ }^{1}$, Takanori Igarashi ${ }^{2}$, \\ Keisuke $\mathrm{Nakao}^{2}$, and Akio Kashimoto ${ }^{2}$ \\ ${ }^{1}$ Graduate School of Science and Engineering, Ritsumeikan University, Japan \\ ${ }^{2}$ Beauty Cosmetic Research Lab, Kao Corporation, Japan \\ chendis.ritsumei.ac.jp
}

\begin{abstract}
We constructed a multi-angle view, illumination and cosmetic facial image database (MaVIC) for quantitative characterization of facial appearance. The facial images in the database are captured from various angles of view and of illumination with a multi-angle image capturing system (MICS). In MaVIC, there are 170 Japanese female's natural facial images and 250 Japanese female's cosmetic facial images. Each subject was photographed in 13 different view-directions under 14 illuminations. Quantitative analysis has been done for the images in MaVIC by the use of principal component analysis (PCA).
\end{abstract}

Keywords: Multi-angle view, multi-angle illumination, cosmetic, facial image database, principal component analysis, opto-chromatic image.

\section{Introduction}

Quantitative characterization of facial appearance is an important issue in many fields and applications including cosmetic foundation design [1,2]. Two of the most important requirements for facial appearance studies are: (1) large database of facial images with multi-angle view, illumination and various types of facial appearance; and (2) a model describing the facial appearance with fewer parameters quantitatively.

To date, many face databases have been constructed for face recognition, face detection and face modeling, such as FERET [3], CMU PIE [4], ORL [5], MITCBCL[6], Yale[7], CAS-PEAL[8], AR[9], UMIST[10], Oulu[11], JAFFE[12] and so on. Especially, CMU PIE and CAS-PEAL are multi-angle view and illumination facial image databases. Unfortunately, since these face database are designed for face recognition and face detection, the image resolution is not enough for quantitative studies of facial appearance. There are also no any variations on facial appearance in CMU PIE or CAS-PEAL. Researchers at Rutgers University constructed a Skin Texture Database [13] with various variations of skin appearance, but it is not a facial image database.

We have constructed a facial image database for quantitative studies of facial appearance. We called this database the KAO-Ritsumeikan Multi-angle View, Illumination and Cosmetic Facial Image Database (MaVIC). The facial images in the 
database are captured from various angles of view and of illumination with a multiangle image capturing system (MICS) [14]. MICS is a system that consists of 20 CCD cameras and 40 flashes that are multi-directionally attached to a hemispherical gantry. In MaVIC, there are 170 Japanese female's natural facial images and 250 Japanese female's cosmetic facial images. Each subject was photographed in 13 different viewing directions under 14 illuminations. There are more than 76000 facial images in MaVIC. The unique features of our database are: (1) the multiangles of view and of illumination are well controlled and multiple-view images under one illumination can be captured simultaneously in one shot; (2) the image resolution is very high (6M pixels) so that the image can also be used as skin texture image; and (3) various types of facial appearances of Japanese females in all ages, which are created by makeup artists, are available.

In order to characterize the opto-chromatic features of the facial appearance, each color facial image in MaVIC is transformed to $\mathrm{L}^{*}$ image and $\mathrm{C}^{*}$ image by the $\mathrm{L} * \mathrm{C} * \mathrm{~h}$ transform, which represent the distribution mapping of brightness and chroma, respectively [15]. Principal Component Analysis (PCA) [16] is performed to L* images and $\mathrm{C} *$ images, respectively in order to find main components contributing to the facial appearance and the dependence on the viewing and illumination directions. Significant difference of PCA coefficients have been found for different facial appearances which are created by makeup artists such as "soft", "wet", "sharp" and "heavy".

The paper is organized as follows: the multi-angle image capture system is described in Section 2; the captured facial images and database MaVIC are described in Section 3; the quantitative analysis are presented in Section 4; finally the conclusion is given in Section 5.

\section{Multi-angle Image Capture System}

In order to capture facial images of a person from multi-angle, we built a multi-angle image capturing system (MICS)[14] in Beauty Cosmetic Research Lab, Kao Corporation. The setup of MICS is shown in Figure 1. MICS is a system that consists of 20 CCD cameras and 40 flashes that are multi-directionally attached to a hemispherical gantry with a radius of $1.5 \mathrm{~m}$. The subject sits in a chair with her head in the center. All cameras and flashes are pointed to the center. The relative locations of cameras and flashes are illustrated in Figure 2. The red one is a camera and the yellow one is a flash. The 20 CCD cameras are each separated by 15 degree. The 40 flashes are also each separated by 15 degree. The 13 cameras among 20 are set in a horizontal plane from full left to full frontal and on to full right. The horizontal plane is in equal height with the subject's head. The 7 other cameras include 3 above the center camera, 2 upper right and 2 upper left (see Figure 2). The images that the 13 cameras in the horizontal plane took from each position are shown in Figure 3. In our system, high resolution $\mathrm{CCD}$ cameras with $6 \mathrm{M}$ pixels are used. So the detailed skin texture can be imaged, which will be shown in Figure 4 later. All of the cameras and flashes are connected to and controlled by a computer. So we can capture the 20 images from different viewing angles simultaneously in one shot. The system is set in a darkroom resulting in a uniform dark background in the image. 


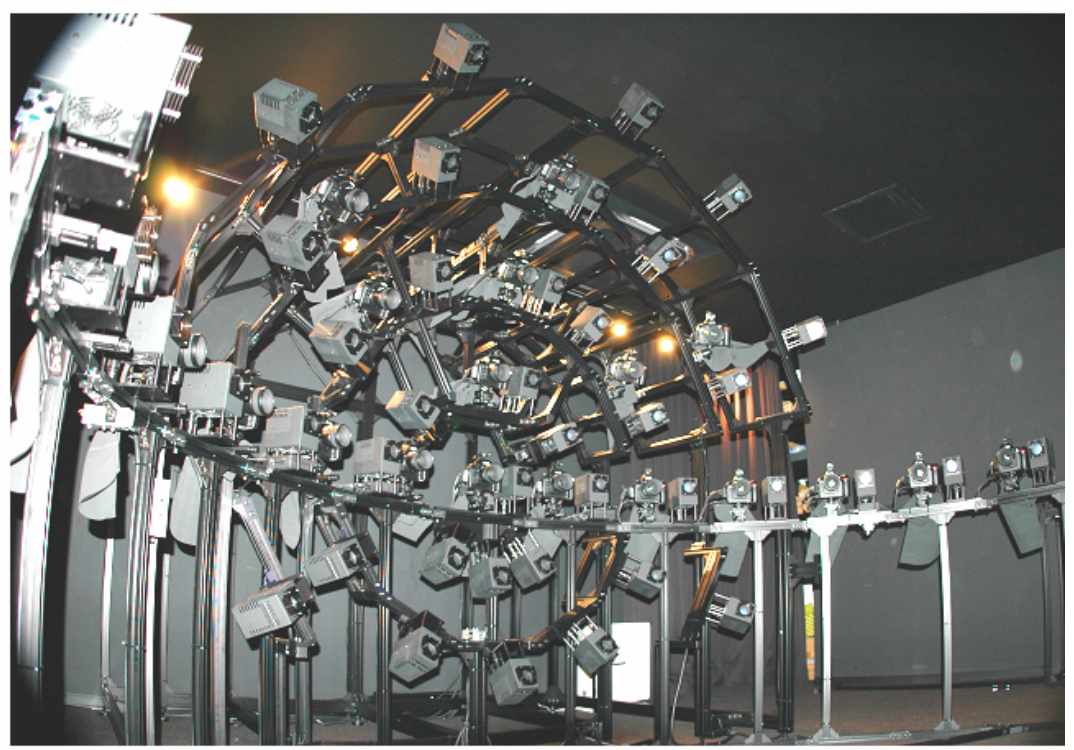

Fig. 1. The setup of MICS

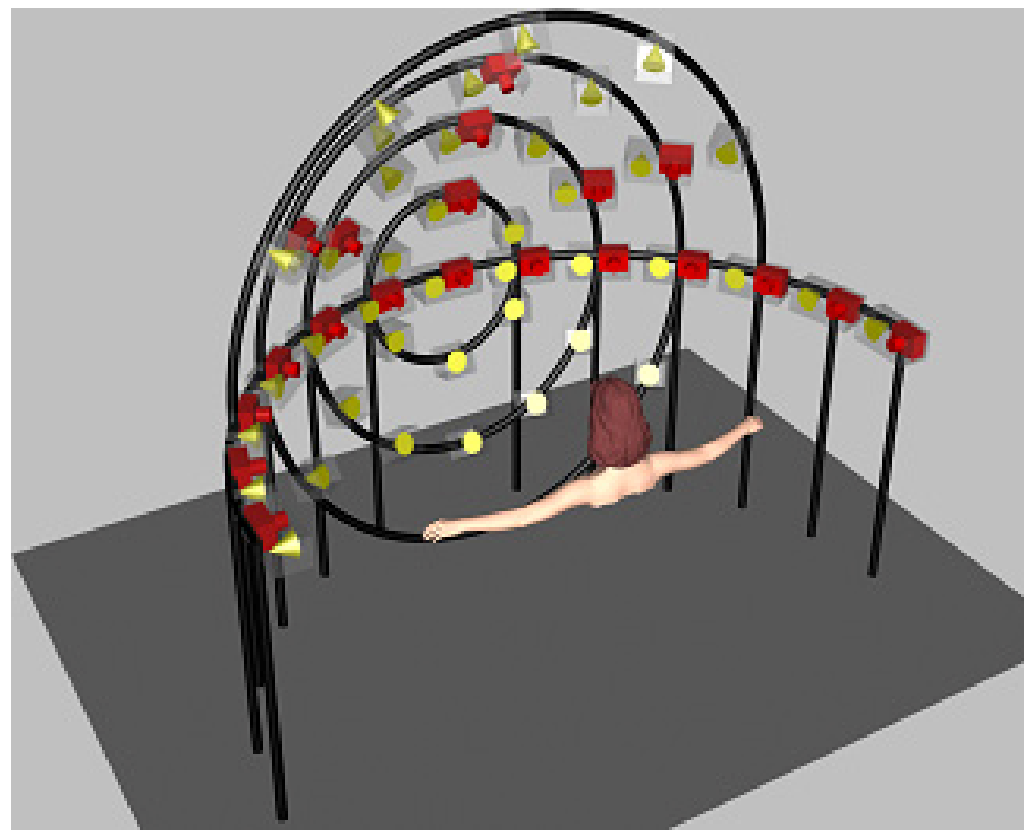

Fig. 2. Configuration of cameras and flashes 


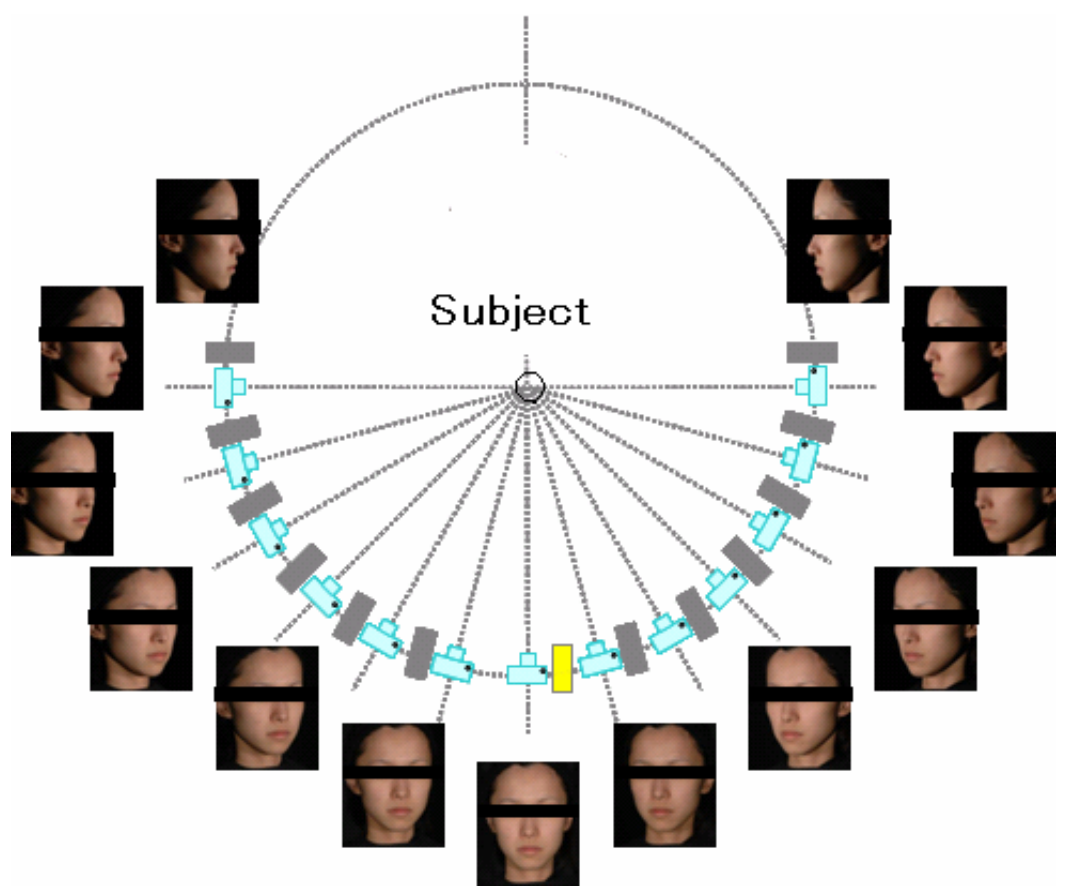

Fig. 3. Multi-angle images of one subject taken by MICS

\section{Facial Images and Database: MaVIC}

By using MICS described in Section 2, we collected more than 76000 images and constructed a facial image database for quantitative studies of facial appearance. We called this database the KAO-Ritsumeikan Multi-angle View, Illumination and Cosmetic Facial Image Database (MaVIC). In MaVIC, there are 170 Japanese female's natural facial images and 250 Japanese female's cosmetic facial images. Each subject was photographed in 13 different viewing directions under 14 illuminations. It should be noted that we only use the cameras and flashes in the horizontal plane to collect the images. Each subject is requested to shut eyes, sit straight and turn forward.

The database consists of three parts: MaVIC1 consisting of 70 Japanese female's natural facial images; MaVIC2 consisting of 100 Japanese female's natural facial images and MaVIC 3 consisting of 250 Japanese female's cosmetic facial images. 100 of 250 subjects in MaVIC3 are the same subjects as MaVIC2. It can be considered as a variation of facial appearance. This is one of unique features of our database. The relationship among MaVIC1, MaVIC2 and MaVIC3 is summarized in Table 1.

The images are 3008x2000 color images. One example is shown in Figure 4(a). Each image is first geometrically normalized. The image is translated so that the eyes are positioned in line. Then the face image is cropped to include only the face with little hair and background. The size of the cropped face image is $800 \times 800$, which is 
Table 1. Contents of MaVIC

\begin{tabular}{cccc}
\hline Subjects & $\begin{array}{c}\text { MaVIC1 } \\
\text { (natural) }\end{array}$ & $\begin{array}{c}\text { MaVIC2 } \\
\text { (natural) }\end{array}$ & $\begin{array}{c}\text { MaVIC3 } \\
\text { (cosmetic) }\end{array}$ \\
\cline { 1 - 1 } 70 & $\circ$ & & 0 \\
100 & & $\circ$ & 0 \\
\hline
\end{tabular}

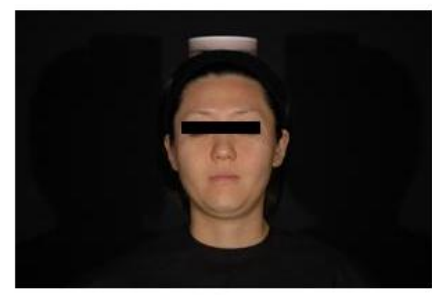

(a)

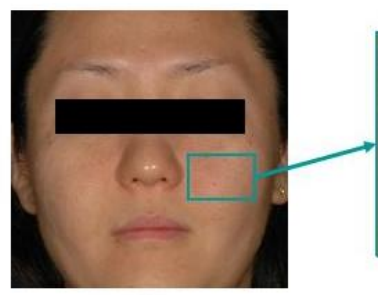

(b)

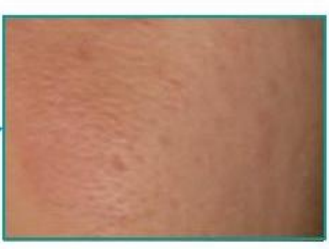

(c)

Fig. 4. (a) Original Image; (b) cropped face image; (c) skin texture image

The examples of multi-angle view and illumination facial images are shown in Figure 5 and four types (heavy, sharp, soft and wet) of cosmetic facial images created by makeup artists are shown in Figure 6.
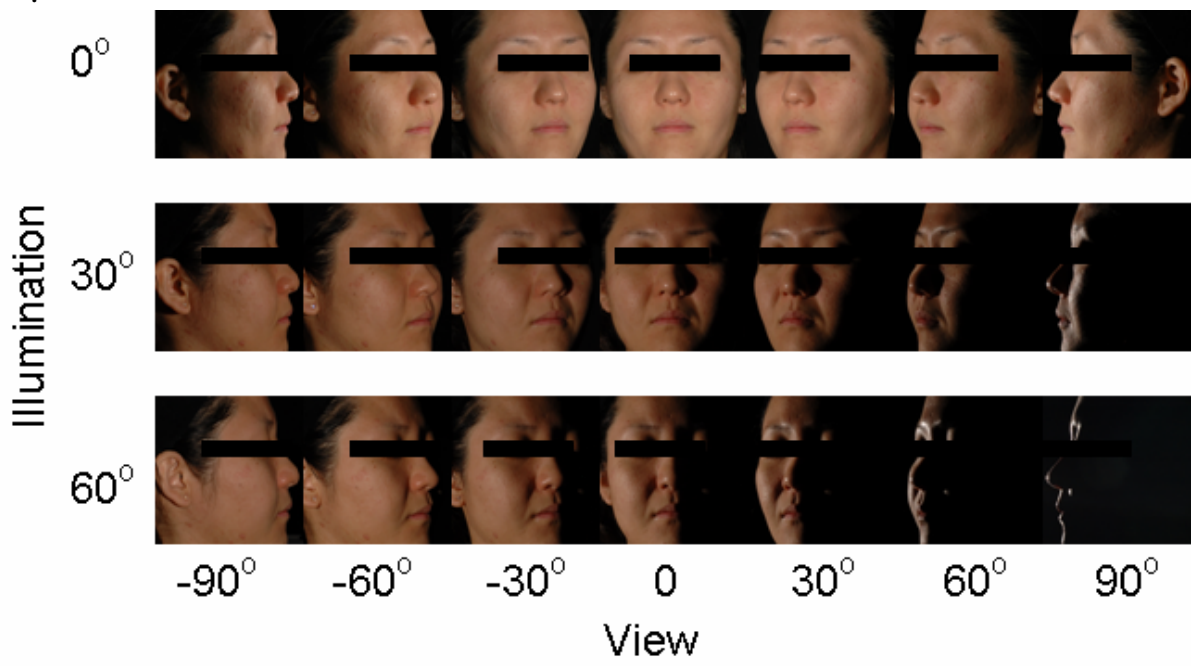

Fig. 5. Multi-angle view and illumination facial images

shown in Figure 4(b). The image resolution of our database is much higher than that of existing databases such as PIE [4], CAS-PEAL[8]. So detailed information about skin texture is also available as shown in Figure 4(c), which is another unique feature of our database. 


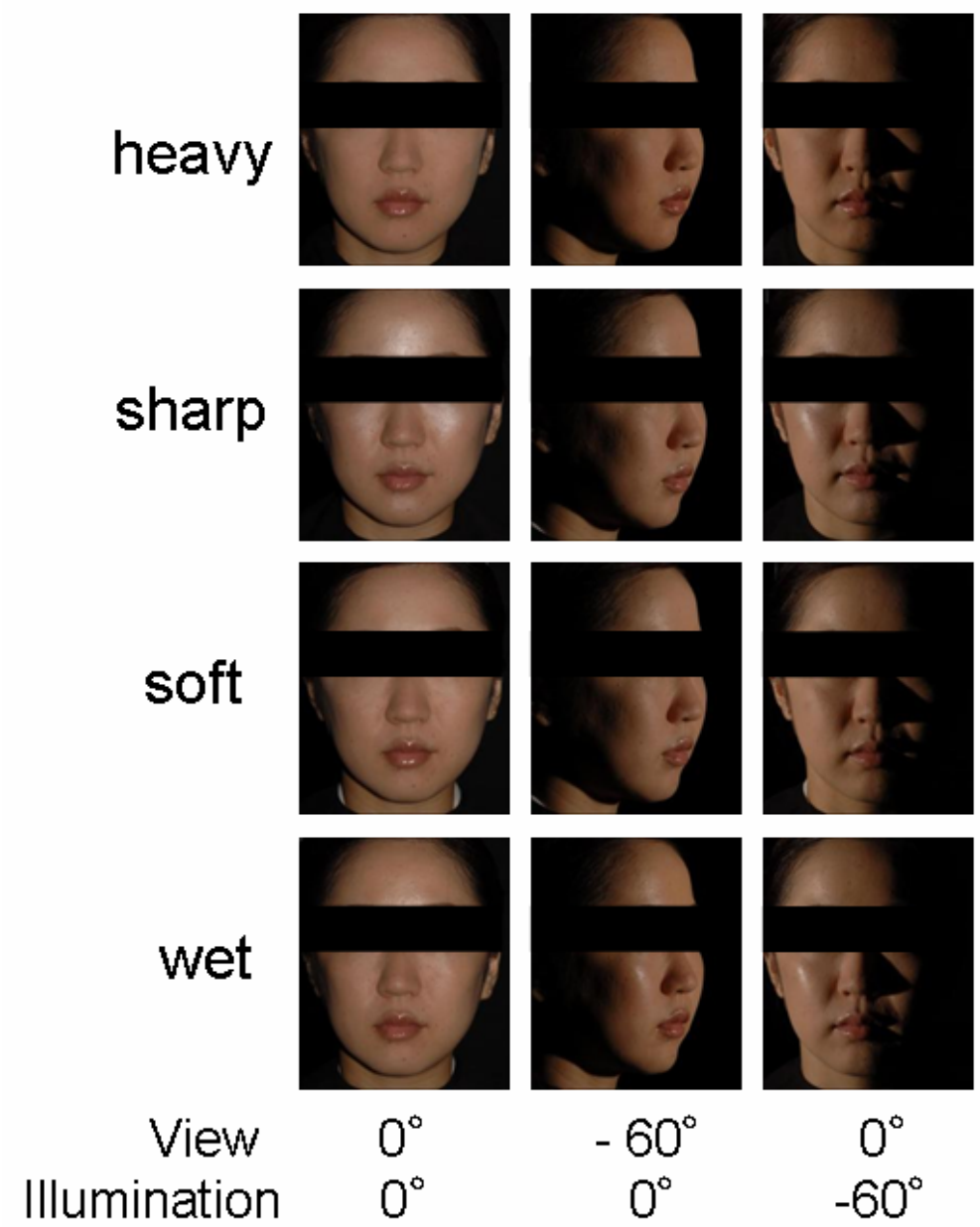

Fig. 6. Four types of cosmetic images created by makeup artists

\section{Quantitative Analysis}

\subsection{Opto-chromatic Images}

In order to characterize the opto-chromatic features of the facial appearance, each color facial image is transformed to $\mathrm{L}^{*}$ image and $\mathrm{C}^{*}$ image by the $\mathrm{L} * \mathrm{C} * \mathrm{~h}$ transform, which represent the distribution mapping of brightness and chroma, respectively [11]. Examples of chromatic distribution maps for different types of facial appearance (Figure 6) created by makeup artists are shown in Figure 7. It can be seen that the facial appearance varied significantly depending on the directions from which they were viewed and illuminated. Specifically, we found that the patterns of the directional variations differed widely depending on the type of facial appearance, and this reflected the opto-chromatic features of their appearances. 


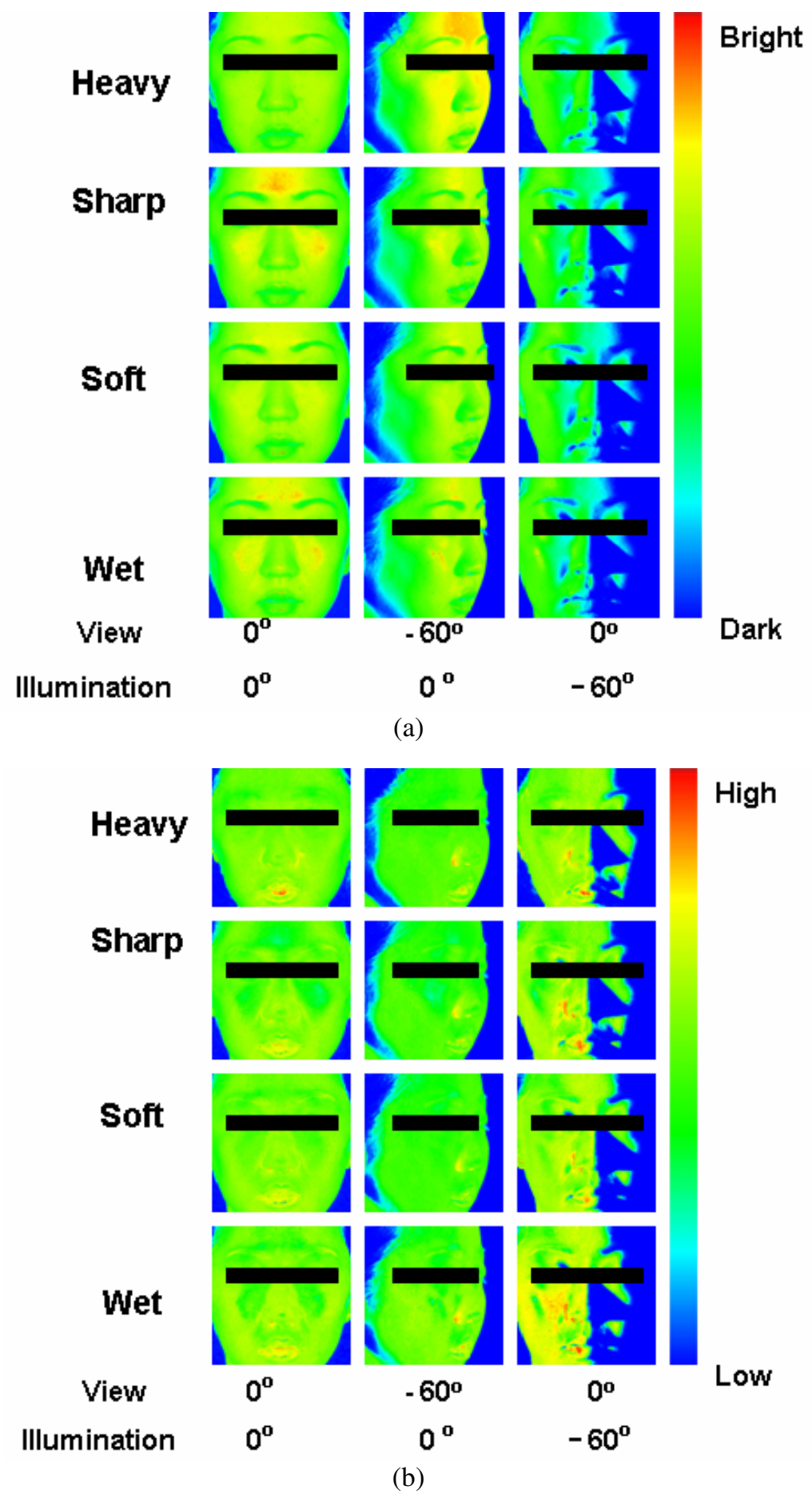

Fig. 7. (a) $\mathrm{L} *$ images; (b) $\mathrm{C} *$ images 


\subsection{PCA Based Subspace Analysis}

Principal Component Analysis (PCA) [16] is performed to $\mathrm{L}^{*}$ images and $\mathrm{C}^{*}$ images, respectively in order to find main components contributing to the facial appearance and the dependence on the viewing and illumination directions.

The facial image or facial appearance $\mathbf{x}$ can be expressed as

$$
\mathbf{x}=s_{0} \mathbf{v}_{0}+s_{1} \mathbf{v}_{1}+\cdots+s_{n} \mathbf{v}_{n}
$$

where $\mathbf{v}_{\mathrm{i}}$ is the basis function and $\mathrm{s}_{i}$ is the coefficient, which can be used as image features or image coding. The basis functions are learned from sample images by PCA. Since the dimension of sample images is large, we use a method called eigenfaces method [12] to calculate the basis functions or eigen subspace.

The 2D facial image is first unfolded into a vector a. Suppose a series of vectors with zero-mean, $\mathbf{a}_{i}, i=1,2, \cdots, M$ and $\sum_{i=1}^{M} \mathbf{a}_{i}=0$, are given. In conventional PCA, the bases of the vector space can be directly obtained by selecting the $J$ leading eigenvectors, $\mathbf{v}_{j}$, of the covariance matrix $\mathbf{C o v}_{1}$, where $\mathbf{C o v}_{1}=\mathbf{A} \cdot \mathbf{A}^{T}$ and $\mathbf{A}=\left[\mathbf{a}_{1}, \mathbf{a}_{2}, \cdots, \mathbf{a}_{M}\right]$.

Since the dimension of $\mathbf{C o v}_{1}$ is determined by the dimension of the vector $\mathbf{a}_{i}$, if the vector has large dimensions, the computing cost for the eigenvectors is huge. In eigenface method [12], instead of calculating the bases directly from $\mathbf{C o v}_{1}$, the eigenvectors $\mathbf{u}_{j}$ is first calculated from $\mathbf{C o v}_{2}=\mathbf{A}^{T} \cdot \mathbf{A}$. Then the basis of the unfolding

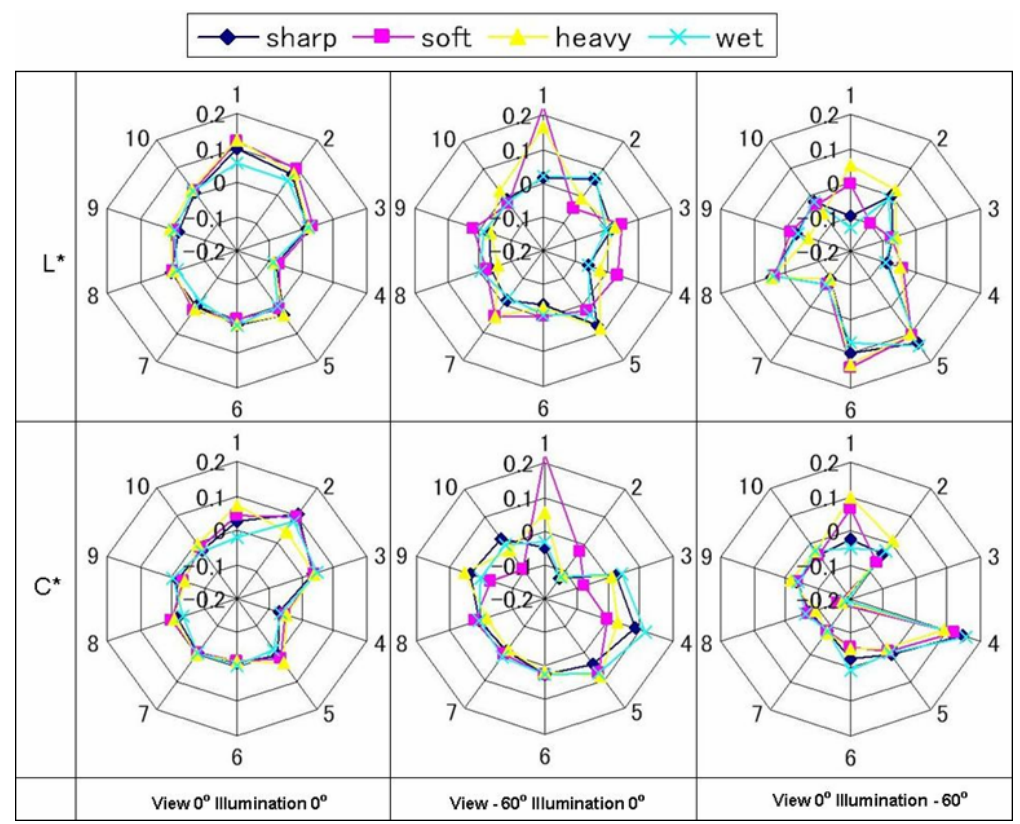

Fig. 8. Comparison of PCA coefficients 
vector space $\mathbf{v}_{j}$ can be calculated by $\mathbf{v}_{j}=\mathbf{A} \cdot \mathbf{u}_{j}$. The dimension of $\mathbf{C o v}_{2}$ is depended on the number of training samples.

We first calculated the $L^{*}$ eigenface (brightness subspace) and $C^{*}$ eigenface (chromatic subspace) of each viewing angle and illumination condition using 300 corresponding $\mathrm{L}^{*}$ facial images and $\mathrm{C}^{*}$ images (150 natural images +150 cosmetic images), respectively. Then we projected the $\mathrm{L}^{*}$ image and $\mathrm{C}^{*}$ image of various types of facial appearance (Figure 6) to the corresponding subspace, and calculated their coefficients. The 10 leading coefficients are plotted in Figure 8. It can be seen that the PCA coefficients are different (especially in the lower mode) for different facial appearances. When the viewing angle and illumination condition are different, the coefficients are also different. That means the PCA coefficients may be used as a quantitative measure of facial appearance. Detailed discussion about the analysis results is beyond the scope of this paper, it will be presented in [15].

\section{Conclusion}

We have constructed a multi-angle view, illumination and cosmetic facial image database (MaVIC), which is only one and useful database for appearance studies. By combining the MaVIC with chromatic distribution mapping and PCA based subspace analysis, we can understand the quantitative characterization of facial appearance, which will be very useful for designing and controlling various types of facial appearance using cosmetic foundations [11]. A more accurate statistical analysis in which all the face shape is normalized by warping is under research.

\section{References}

1. Igarashi, T., et al.: The Appearance of Human Skin: A Survey. Foundations and Trends in Computer Graphics and Visions 3, 1-95 (2007)

2. Tsumura, N., et al.: Image-based skin color and texture analysis/synthesis by extracting hemoglobin and melanin information in the skin. ACM Transactions on Graphics 22, 770 779 (2003)

3. Phillips, P.J., et al.: The FERET Evaluation Methodology for Face-Recognition Algorithms. IEEE Trans. Pattern Analysis and Machine Intelligence 22, 1090-1104 (2000)

4. Sim, T., Baker, S., Bsat, M.: The CMU Pose, Illumination, and Expression Database. IEEE Trans. Pattern Analysis and Machine Intelligence 25, 1615-1618 (2003)

5. http://www.cl.cam.ac.uk/research/dtg/attarchive/ facedatabase.html

6. http://cbcl.mit.edu/software-datasets/heisele/ facerecognitiondatabase.html

7. http://cvc.yale.edu/projects/yalefaces/yalefaces.html

8. Gao, W., et al.: The CAS-PEAL Large-Scale Chinese Face Database and Baseline Evaluation. IEEE Trans. on System Man, and Cybernetics (Part A) 38, 149-161 (2008)

9. http://rvl1.ecn.purdue.edu/ aleix/aleix_face_DB.html

10. http://images.ee.umist.ac.uk/danny/database.html

11. http://www.ee.oulu.fi/research/imag/color/pbfd.html 
12. http://www.irc.atr.jp/Emlyons/jaffe.html

13. http://www.caip.rutgers.edu/rutgers_texture/

14. Igarashi, T.: Recent technical trends on cosmetic foundations. Fragrance Journal 34, 17-28 (2006)

15. Igarashi, T., et al.: Face Designing: Technology to Control Facial Appearance Based on Multi-angle Image Analysis and Electromagnetic Simulation. In: Proc. IFSCC 2008 (to be published, 2008)

16. Turk, M., Pentland: Eigenfaces for Recognition. Journal of Cognitive Neuroscience 3(1), 71-86 (1991) 